\title{
Technical note: An empirical method for absolute calibration of coccolith thickness
}

\author{
Saúl González-Lemos ${ }^{1, *}$, José Guitián ${ }^{2, *}$, Miguel-Ángel Fuertes ${ }^{3}$, José-Abel Flores ${ }^{3}$, and Heather M. Stoll ${ }^{1,2}$ \\ ${ }^{1}$ Department of Geology, University of Oviedo, Oviedo, 33005, Spain \\ ${ }^{2}$ Department of Earth Sciences, ETH Zurich, Zurich, 8092, Switzerland \\ ${ }^{3}$ Department of Geology, University of Salamanca, Salamanca, 37008, Spain \\ *These authors contributed equally to this work.
}

Correspondence: Saúl González-Lemos (saulgonzalezlemos@gmail.com) and Heather M. Stoll (heather.stoll@erdw.ethz.ch)

Received: 12 June 2017 - Discussion started: 1 August 2017

Revised: 20 December 2017 - Accepted: 5 January 2018 - Published: 22 February 2018

\begin{abstract}
As major calcifiers in the open ocean, coccolithophores play a key role in the marine carbon cycle. Because they may be sensitive to changing $\mathrm{CO}_{2}$ and ocean acidification, there is significant interest in quantifying past and present variations in their cellular calcification by quantifying the thickness of the coccoliths or calcite plates that cover their cells. Polarized light microscopy has emerged as a key tool for quantifying the thickness of these calcite plates, but the reproducibility and accuracy of such determinations has been limited by the absence of suitable calibration materials in the thickness range of coccoliths $(0-4 \mu \mathrm{m})$. Here, we describe the fabrication of a calcite wedge with a constant slope over this thickness range, and the independent determination of calcite thickness along the wedge profile. We show how the calcite wedge provides more robust calibrations in the 0 to $1.55 \mu \mathrm{m}$ range than previous approaches using rhabdoliths. We show the particular advantages of the calcite wedge approach for developing equations to relate thickness to the interference colors that arise in calcite in the thickness range between 1.55 and $4 \mu \mathrm{m}$. The calcite wedge approach can be applied to develop equations relevant to the particular light spectra and intensity of any polarized light microscope system and could significantly improve inter-laboratory data comparability.
\end{abstract}

\section{Introduction}

The calcification by coccolithophorid algae represents a major source of $\mathrm{CaCO}_{3}$ production in the open oceans (Siesser, 1993). This calcite is believed to be key to ocean biogeochemical cycles, as it increases deep export of organic carbon through the ballasting of aggregates (Abrantes et al., 2002; Armstrong et al., 2002, 2009; Bárcena et al., 2004; Berger et al., 1989; Engel et al., 2009; Iversen and Ploug, 2010; Milliman, 1993; Ridgwell and Zeebe, 2005; Ziveri et al., 2007). Consequently, there is interest in the response of coccolithophorid calcification to changes in ocean productivity and carbon systems. To move beyond the responses of calcification in clonal cultures in short-duration experiments, recent efforts have focused on characterizing the variations in coccolithophorid calcification from measurements of the thickness of coccoliths, in populations from the water column and ocean sediments. Such measurements are effectively made using images of coccoliths taken by polarized light microscopy, for which the interference color varies with the thickness of the calcite in the coccolith (Beaufort, 2005). Recent advances using circular polarized light (Bollmann, 2014; Fuertes et al., 2014) permit such calculations with a single image rather than a composite at several orientations as was done in previous approaches (Beaufort et al., 2014).

Until recently, coccolith thickness measurements have been limited to thicknesses less than $1.55 \mu \mathrm{m}$, for which birefringence remains in grayscale tones. Beaufort et al. (2014) suggested that measurements might be extended to $4.5 \mu \mathrm{m}$ thickness by employing variations in the colored birefrin- 
gence range using a set of equations in HSL (hue, saturation, lightness) color system.

While there is a clear theoretical dependence of interference color on calcite thickness, the particular grayscale or color value obtained for a given calcite particle also depends on the microscope configuration, light intensity, and spectra of the light employed at the time of measurement. Even for a given microscope system, this relationship changes over time due to bulb aging and microscope settings, so a daily calibration is required. However, this calibration is challenging due to the absence of reference materials, for which the observed interference color on a given day may be related to a known thickness. The situation becomes even more complex for coccoliths thicker than $1.55 \mu \mathrm{m}$, as there is no longer a single bivariate theoretical curve between grayscale and thickness; rather representation of thickness depends on colors registered as three parameters (e.g. red, green, blue, RGB; or hue, saturation, value, HSV; or HSL). Beaufort et al. (2014) proposed a set of equations to relate HSL color values to thickness in the range of 1.55 to $4.5 \mu \mathrm{m}$, but these particular equations may be specific to particular light settings and manufacturer configurations of a particular microscope and thus should not be universally applied.

Here we discuss a calibration option relevant for the grayscale and color region, and which readily allows each user to establish relevant equations for his or her own microscope system, which may be modified to account for effects such as bulb aging and light spectra. We describe the preparation and use of a calcite wedge for which thickness can be independently constrained, suitable as a daily calibration material for both grayscale and color scale. In this work, following previous studies (Bollmann, 2014; Craig, 1961; Fuertes et al., 2014), we employ circular polarized light (Bass, 2009; Frohlich, 1986; Hecht, 2002; Higgins, 2010) so that our interference colors are independent of the calcite $c$-axis orientation and coccoliths are viewed without extinction patterns. With this calcite wedge, the user can calibrate microscope-specific equations relating color to thickness. A calcite wedge is used because it permits direct comparison between interference colors in the wedge and in the coccoliths, because both are made of calcite with the same birefringence.

\section{Background on use of the polarized circular light and details of previous calibrations}

Anisotropic minerals, such as the calcite comprising coccolithophores, modulate the vibration direction of light passing through them. When light passes through an anisotropic (birefringent) mineral, the light is split into two rays of different velocity. The retardation of the slow ray relative to the fast ray increases with the increasing thickness of the mineral. For a given mineral, the equation relating thickness $(t)$ and retardation $(r)$ is given by Eq. (1) (Delly, 2003):

$$
t=\frac{r}{b \times 1000},
$$

where the birefringence $(b)$ is a property of the mineral lattice $(0.172$ for calcite). Optically, this retardation is manifest by the appearance of interference colors when the light rays emerging from the mineral recombine after passing through a second polarizing filter. For a given mineral orientation, progressive degrees of retardation produce a sequence of interference colors first defined in the Michel-Lévy chart, recently revised by Sørensen (2013). In the case of calcite, these range from black through gray to white at a thickness of $1.55 \mu \mathrm{m}$, then to yellow, red, and blue as thicknesses progressively increases up to $4 \mu \mathrm{m}$.

Several approaches have been used previously to ascertain coccolith thickness from measured grayscale values of coccoliths in the thickness range from 0 to $1.55 \mu \mathrm{m}$. Beaufort (2005) was first to use smear slides with a known weight of calcite particles to construct an empirical gray-value calibration curve. O'Dea (2014) applied a theoretical sigmoidal relationship between grayscale and thickness. Another common approach has been to assume a cylindrical shape of rods of common nannofossil genera Rhabdosphaera clavigera, so that rhabdolith diameter is taken to be the known thickness, and the grayscale value corresponding to this thickness can be used for an empirical calibration or for pinning tie points to a theoretical sigmoidal calibration (Beaufort and Dollfus, 2004; Beaufort et al., 2014; Fuertes et al., 2014). Alternatively, Bollmann (2014) employed polymer sheets which produce a specified degree of retardation. Lochte (2014) tested two calibration techniques proposed by Beaufort et al. (2014) and Bollmann (2014) to compare coccolith weight estimates of cultured single clones of Gephyrocapsa oceanica, encountering some challenges with Rhabdosphaera calibration.

Our method with a calcite wedge permits calibration continuously over the thickness range from 0 to $4 \mu \mathrm{m}$, contributing to reproducible and accurate thickness measurements of a wide range of genera of modern and ancient coccoliths.

\section{Preparation and validation of calcite wedge}

\subsection{Manufacture of calcite wedge thin sections}

A natural calcite crystal of Iceland spar was used to produce several calcite wedges. A plane free of fissures and defects was selected and a small rectangular prism was cut using a water-cooled diamond saw. This fragment was abraded with carborundum-water slurry, polished, and glued with epoxy resin to a glass slide, using pressure to minimize bubble formation between the calcite and glass and heat to accelerate epoxy curing. The calcite was then cut to $1 \mathrm{~mm}$ thickness and further abraded to attain a $100 \mu \mathrm{m}$ thickness. A wedge was attained on one border of the calcite through a final manual abrasion step, by applying greater pressure on one edge of 
(a)
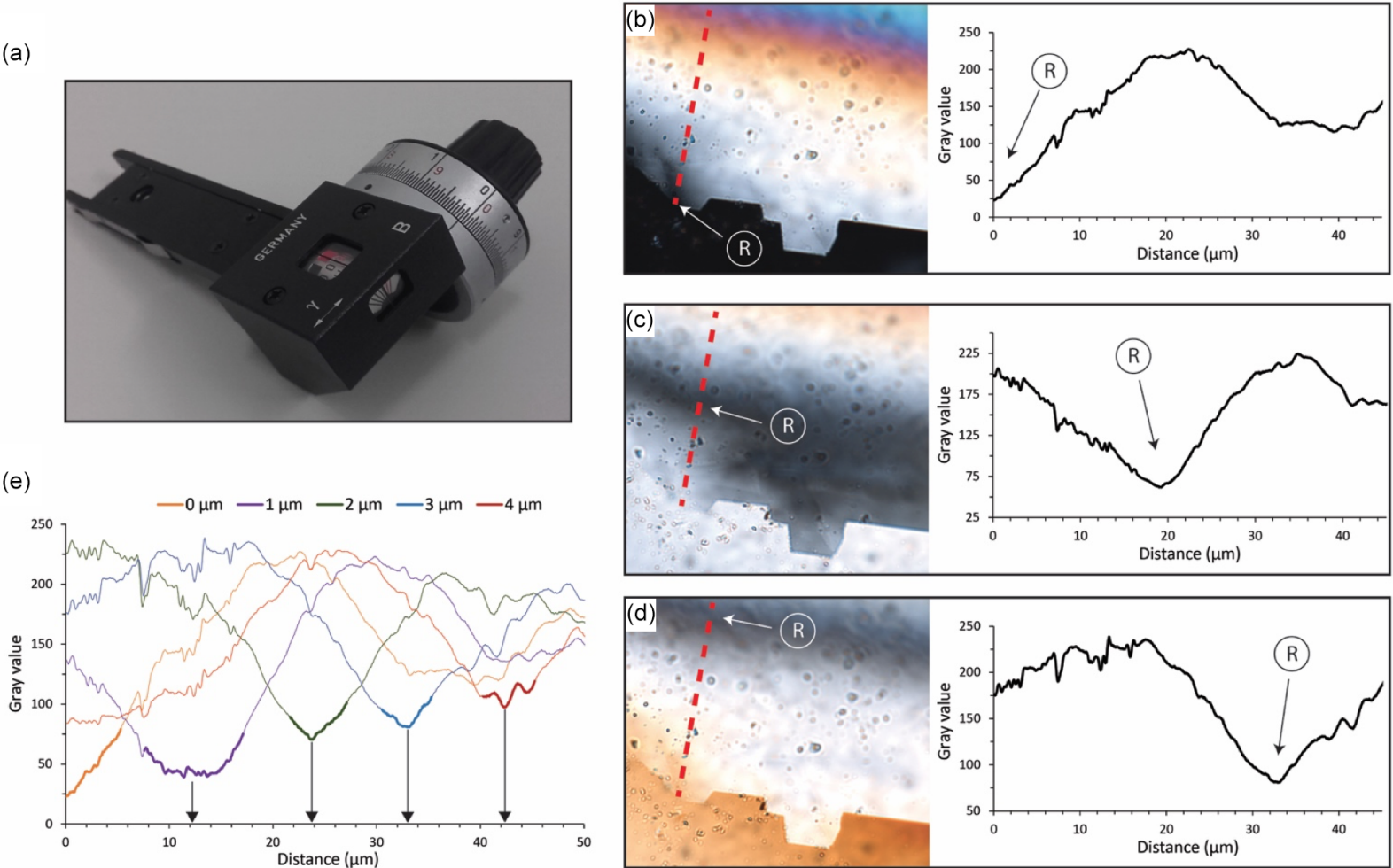

Figure 1. Technique for quantifying wedge thickness. (a) Tilting compensator B 0-5 Lambda. (b-d) Schematic succession of images showing the migration of the compensation point under along the calcite wedge (OVD-W1) linearly polarized light with successive rotations of the compensator. The minimum grayscale value in each image corresponds to the full compensation of interference colors at that point, and is designated by an "R" in each image. The blue tone produced by partial compensation of interference colors leads to second local minima in grayscale on either side of the full compensation. (e) Full grayscale profiles along the wedge with compensator rotation equivalent to four selected thicknesses of calcite. Arrows show the distance of maximum compensation for each tilting compensator rotation, and denote the distance on the OVD-W1 wedge corresponding to that thickness. Data in (e) were collected on a Zeiss Axio Scope and were used for quantitative calibration of the wedge. Light intensity was saturated at a calcite thickness of $1.55 \mu \mathrm{m}$.

the slide to accentuate the removal rate of material. During this final abrasion, the edges of the slide were evaluated using a microscope to verify when the borders enter into the first order interference colors corresponding to the thickness range from 0 to $4 \mu \mathrm{m}(0-550 \mathrm{~nm}$ retardation).

Because calcite fractures easily at thicknesses less than $10 \mu \mathrm{m}$, only a small portion of the slide - one or more zones on the edge of the calcite - forms a wedge in the relevant thickness range. The wedge which featured the least number of imperfections in the calcite (e.g., fractures, bubbles, or polishing grit; abrupt thickness changes due to breaks on cleavage planes) was adopted for calibration. We produced multiple slides of Iceland spar prepared in an identical way and identified two as providing an acceptable area with a wedge in the desired thickness range in one part of the slide (Fig. 1). The wedge was protected with a coverslip adhered with Canada balsam, in the same way as the coccolith slides are prepared, avoiding differences in the light absorption among them.
Despite optimal precautions taken against the generation of particles or bubbles, nonetheless some of these were present and generate halos which were visible in highresolution images. For work in the grayscale range, we used a wedge produced at the University of Oviedo (OVD-W1). For work in the color zone, we employed a wedge produced at ETH Zurich (ETH-W2) which had fewer defects in the wedge in the transition to colors.

\subsection{Microscope method}

Digital images were obtained on two similarly configured microscope systems. All grayscale work was conducted at the University of Salamanca using a Nikon Eclipse LV100 POL microscope with circularly polarized light, equipped with a Nikon Plan Fluor 100X/1.30 oil OFN25 DIC H/N2 objective, a universal condenser with numerical aperture of 0.9 and a Nikon digital camera DS-Fi1. The camera resolution is $1920 \times 1280$ pixels, giving a pixel resolution of $0.035 \mu \mathrm{m}$. For color work, images were obtained at ETH Zurich using a Zeiss Axio Scope HAL100 POL microscope with circularly 
(a)

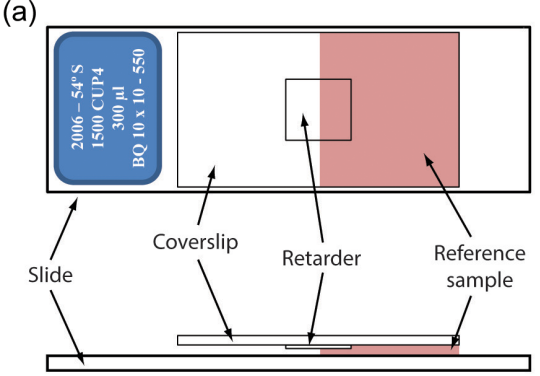

(d)

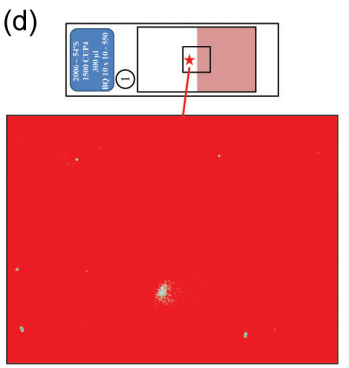

(b)

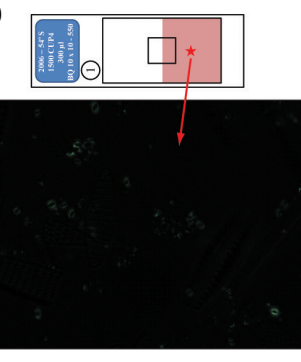

(c)

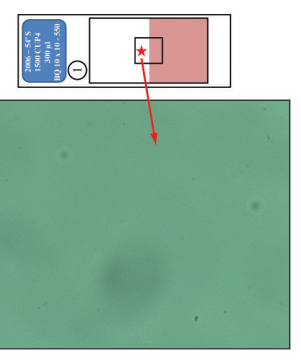

(e)

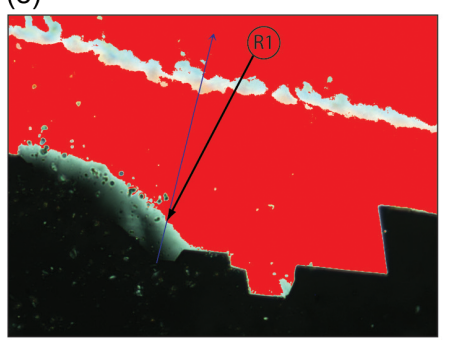

(f)

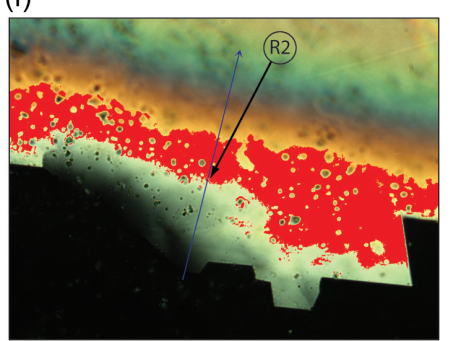

Figure 2. Technique for validating wedge thickness at two tie points. (a) Distribute a retarder polymer and reference sample on a slide. Only half of the retarder is covered by the reference sample. (b) Focus the coccoliths of the reference sample, (c) move to the sample-free part on the retarder polymer, and (d) increase the light intensity until polymer saturation (the image acquisition software indicates the saturated area in red). (e-f) Wedge calcite saturated under the same conditions as the retarders 1 and 2, respectively. The arrows (R1, R2) indicate the points where the thickness has been calculated.

polarized light, equipped with a Zeiss Plan-APOCHROMAT 100x/1.4 Oil objective, a universal condenser with numerical aperture of 0.9 and a Zeiss Axiocam 506 Color. The camera resolution was set to $2560 \times 1920$ pixels, resulting in a pixel resolution of $0.0454 \mu \mathrm{m}$. In all of these microscopes, for circular polarization, two $\lambda / 4$ retardation plates were placed in the microscope, one located between the lower linear polarizer and the condenser and the other below the upper linear polarizer (both at an angle of $45^{\circ}$ relative to it) to transform the linearly polarized light into circularly polarized light (Fuertes et al., 2014). The microscope was turned on for 30-60 min to warm up and stabilize the light conditions before any images were captured. On the Nikon, images were collected using the software specific to the camera "Nis-Elements BR". On the Zeiss microscopes, images were collected using "Zeiss Zen 2.3 (blue edition)" imaging software. For work limited to grayscale range, the light intensity was adjusted to give an optimum range in grayscale values for the materials being quantified. For work including the color range, the light intensity was adjusted to attain saturation at a calcite thickness of $1.55 \mu \mathrm{m}$.

\subsection{Independent determination of thickness of the calcite wedge using a tilting compensator}

To determine the thickness more continuously along the wedge, a tilting compensator B 0-5 Lambda, $6 \times 20$ (D) from Zeiss (Fig. 1a) was used. This compensator consists of a piece of uniaxial birefringent material (magnesium fluoride) cut normal to the optical axis that can be tilted about an axis parallel to the sample (in our case, the calcite wedge). The compensator was used under plane-polarized light, and progressive rotation of the compensator about the horizontal axis generates a decrease in the retardation of the rays emerging from the observed calcite wedge. The tilting angle was read accurately from a calibrated micrometer drum and the corresponding decrease in retardation can be read from the corresponding tables supplied with the compensator. The reported accuracy of the instrument is ca. $\pm 2.5-8 \mathrm{~nm}$ of variation in optical path difference $(\triangle \mathrm{OPD})$. For the measurement of our calcite wedge, at maximum light intensity position, we have rotated the compensator in increments corresponding to $0.25 \mu \mathrm{m}$, capturing a digital image at each increment to identify the distance along the wedge experiencing full compensation (negligible interference = black) (Fig. 1b). At the lowest rotation angle, the small decrease in retardation decreases interference colors only slightly, so the zone of full compensation (negligible interference $=$ black) appears on calcite near the edge of the slide (Fig. 1c). With increasing rotation angles, the decrease in retardation produces a stronger decrease in interference colors, so the zone of negligible interference (black) moves progressively up thicker parts of the calcite wedge towards the interior of the slide (Fig. 1c-e). Small variations in the slope of the calcite profile cause different widths of the dark full compensation zone. Over a series of 16 incremental advances in angle, we quantified the location of the compensation point (Fig. 1e) to derive the re- 
lationship between thickness and distance from the edge of the wedge.

\subsection{Validation of the thickness using polymer retarders}

To validate the thickness profile obtained from the rotating compensator, we have used high-precision plastic polymer retarders from Meadowlark Optics with a wavelength of $550 \mathrm{~nm}$ and a retardation of 136.6 and $274.8 \mathrm{~nm}$, which give a retardation equivalent to calcite with thickness of 0.79 and 1.59 , respectively. Each was mounted in Canada balsam in separate glass slides. On half of each retarder (Fig. 2a) we decanted a suspension of modern coccoliths using the technique of Flores and Sierro (1997). This provided an initial focus point for each slide (Fig. 2b). After focusing, we moved to an area of the retarder without the coccoliths (Fig. 2c) and increased the light intensity until we reached saturation on the polymer (Fig. 2d). Without changing conditions, we placed the calcite wedge under the microscope, precisely focused, and the digitally captured image allowed the software to map light saturation (defined as a gray level value of 255 for a white pixel using an 8-bit camera). The lowest point on the wedge at which saturation is attained corresponds to the thickness of the calcite wedge with a retardation identical to that of the plastic polymer (Fig. 2e). We repeated the procedure with the other polymer (Fig. 2f). Using the known birefringence of calcite and the known retardation of each polymer, we employed Eq. (1) to estimate the thickness of the calcite at each of these two points.

\subsection{Thickness profile of predefined wedge}

For subsequent calibration of the relationship between interference color and thickness, we have defined a working profile across each wedge, referenced in our image analysis routine (ImageJ-Fiji). Along this profile in OVD-W1, the wedge reaches a $4 \mu \mathrm{m}$ thickness at a distance of $42 \mu \mathrm{m}$; in ETH-W2 the $4 \mu \mathrm{m}$ thickness is reached at a distance of $28 \mu \mathrm{m}$ (Fig. 3). The validation points from the retarder polymers fall along the line of thickness calculated from the tilting compensator. All subsequent calibration efforts describe the relationship between interference colors and thickness along these fixed profiles.

\section{Application to thickness measurements in the grayscale range}

Over the thickness range from 0 to $1.55 \mu \mathrm{m}$ the calcite wedge exhibits the expected increase in grayscale, with the expected sigmoidal tail at the lowest thickness values (Fig. 4a). The exact grayscale value for a given thickness is dependent on the light setting and exposure times, but for a given setting it is highly reproducible day to day (Fig. 4a). For work limited to the grayscale range, the light intensity was adjusted to give an optimum range in grayscale values for the materials being quantified.

For measurements of coccolith thickness, optimal sensitivity is obtained when the light setting is adjusted for the range of thicknesses in the samples of interest, for example, higher light and exposure times for samples with very thin coccoliths. This is particularly useful with 8-bit cameras which have a lower range of definition than 14 or 16-bit cameras. By matching the light and exposure settings used for a set of samples with those used to capture an image of the calcite wedge, it is possible to develop a robust calibration between grayscale and thickness under any illumination parameters. For routine calibrations of grayscale to thickness, we select 10 points along the wedge profile, with known thickness established as in Fig. 3.

\subsection{Example application of the calibration system to cultured coccoliths}

We have used the calcite wedge to calibrate coccolith thickness measurements in coccoliths produced by eight strains of coccoliths in controlled laboratory culture conditions (as described in Bolton et al., 2016). Image processing was carried out by the C-Calcita MATLAB routine described by Fuertes et al. (2014). The C-Calcita routine permits either a linear or sigmoidal regression between grayscale and thickness. The linear option, with a zero intercept, has been employed in previous studies (Beaufort, 2005) and was employed in our original processing of the culture sample images (Bolton et al., 2016). Recent updating of the MichelLévy curve (Sørensen, 2013) suggests that in the first order interference range the grayscale thickness relationship is better represented with a sigmoidal curve, an approach adopted by recent coccolith thickness studies (Beaufort et al., 2014; O'Dea et al., 2014). An updated version of the C-Calcita routine now permits sigmoidal calibration using an arcsine function to fit the calibration points made from the calcite wedge.

For the same image sets and calibration points from the calcite wedge, we have compared the thickness estimated from the linear vs. sigmoidal calibration slopes for three of the culture samples. Given that a high proportion of the coccolith has a very low thickness and falls within the sigmoidal part of the calibration relationship, the sigmoidal calibration yields average thickness estimates for coccoliths which are nearly twice those attained with a linear calibration (Fig. 4b, c). For thicker coccoliths, where a greater percentage of the individual coccolith falls within the linear portion of the sigmoidal curve, the difference is not expected to be as significant.

\subsection{Grayscale vs thickness relationship in the calcite wedge and Rhabdosphaera clavigera}

The relationship between grayscale and thickness in the calcite wedge was compared with that of $R$. clavigera nan- 


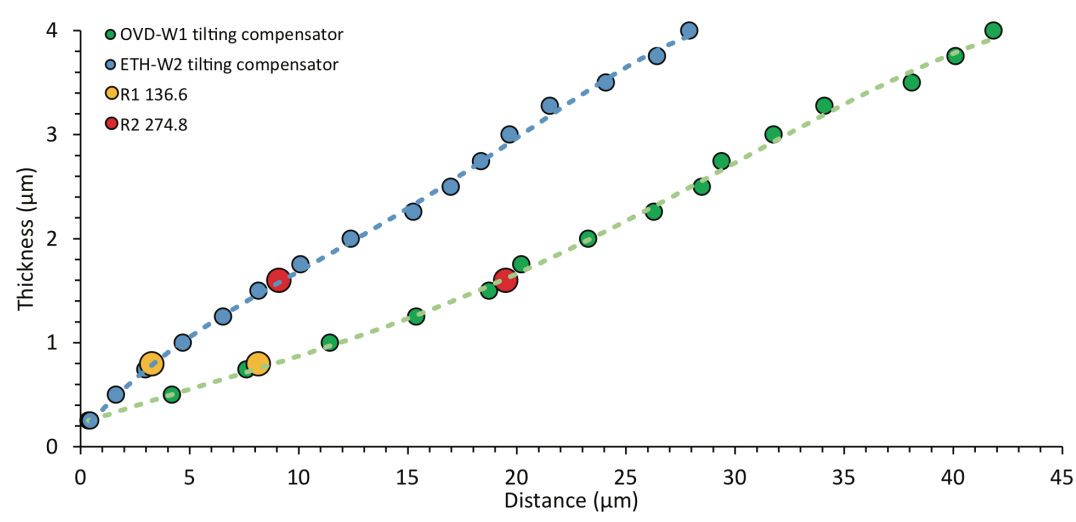

Figure 3. Profile of the calcite wedges OVD-W1 and ETH-W2, as defined by the tilting compensator and the tie points established from the thickness-interference color relationship of the two polymer retarders (R1 and R2).
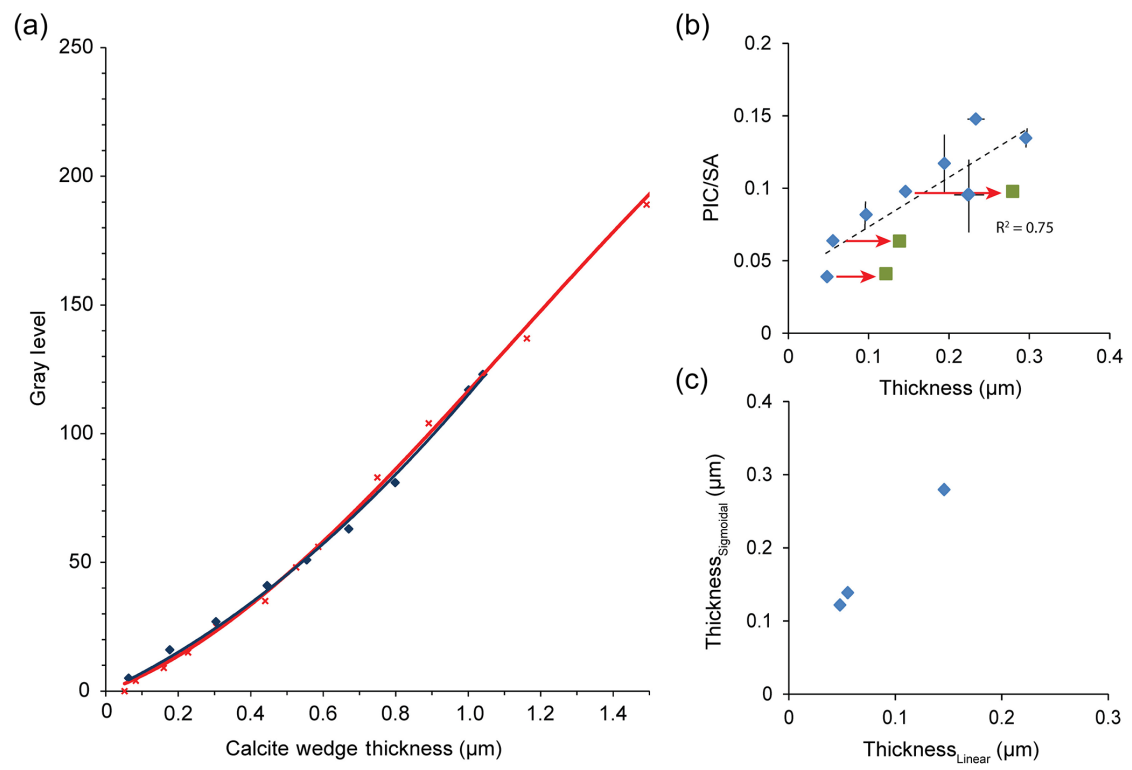

Figure 4. (a) Gray level vs. calcite wedge thickness on images taken with an 8-bit camera. The wedge shows the expected sigmoidal shape at low thicknesses. The reproducibility of the calcite wedge, on images taken under very similar microscopic conditions and light intensity on different moments, is very consistent. The blue line represents a calibration carried out on 29 October 2014, while the red line represents a calibration carried out on 15 July 2015. (b) Comparison of Emiliania huxleyi coccolith thickness estimates using linear and sigmoidal calibration forms. The graphic shows the relationship between cellular calcification (calcite per cell surface area) and coccolith thickness using linear calibration (blue symbols) and sigmoidal calibration (green symbols, connected by red arrows to the equivalent sample measured with linear calibration). (c) Comparison of linear and sigmoidal calibration for three populations of coccoliths from culture.

noliths, applying the conventional assumption that they are cylindrical rods and therefore the diameter is equivalent to the thickness. A total of 15 rhabdoliths were measured under identical light and exposure conditions. After subtracting the background gray level from the image, 10 measurements of thickness and its corresponding gray level at different points were made for each rhabdolith.

For the same estimated thickness, the gray level values show great variation among the chosen rhabdoliths. The regression slopes between estimated thickness and grayscale for each rhabdolith show a large range (Fig. 5a), although if each is forced to pass through the coordinate origin (a premise for applying a calibration), the slopes converge slightly (Fig. 5b). For the same microscope conditions, the individual rhabdolith thickness-grayscale coordinates fall significantly above and below the relationship defined by the calcite wedge (Fig. 5b). The relative standard deviation of 40 to $50 \%$ on the regression slopes from different rhabdoliths is very large compared to the range of thicknesses among different coccolith populations from culture. Measurement of the same rhabdolith for calibration for every session would ensure internally reproducible thickness measure- 

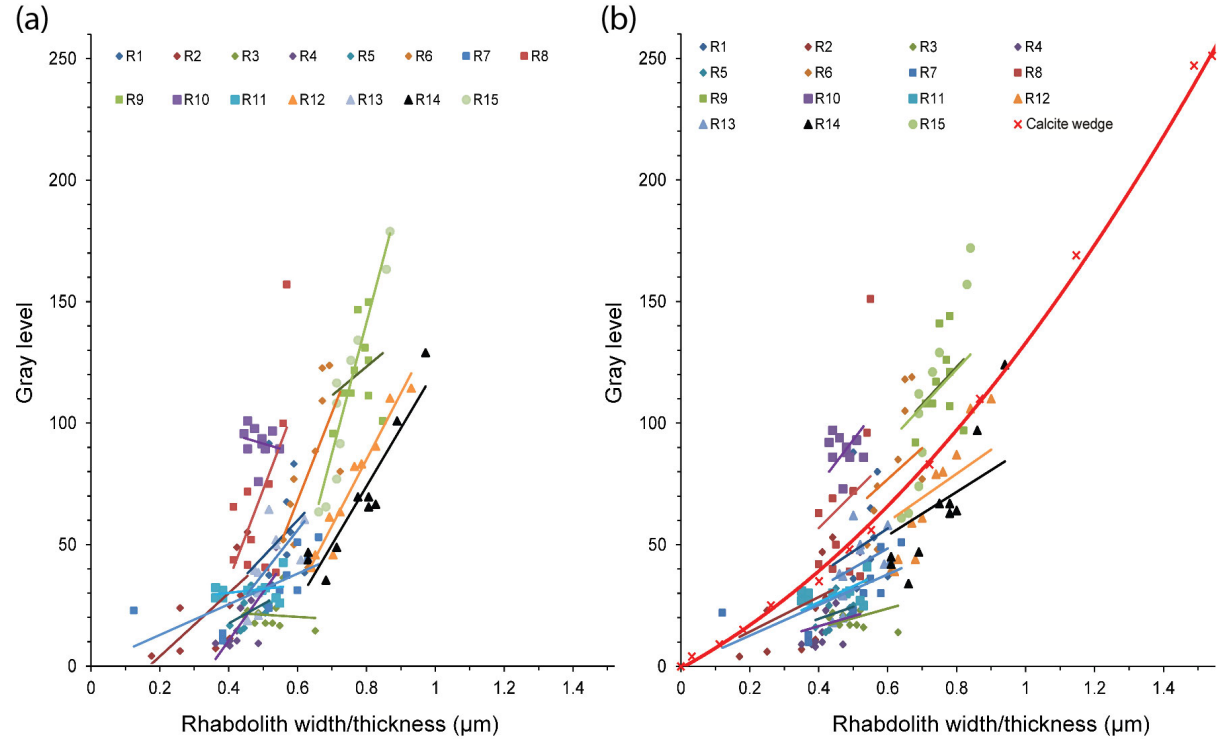

Figure 5. Relationship between grayscale and thickness for 15 individual Rhabdosphaera clavigera rods (R1 to R15), and thickness for the calcite wedge (red line) measured under identical light conditions. The rhabdolith thickness is assumed to the diameter of the rod at the point of grayscale measurement. (a) Natural trend of different rhabdoliths. (b) Rhabdoliths forced to pass for the coordinate origin (requirement for the calibration software). The calibration values for sigmoidal calibration range from a minimum of 0.0024 to a maximum of 0.0113 , with a mean of 0.0059 and standard deviation of 0.0024 . The calibration values for linear calibration range from 0.054 to 0.0252 , with a mean of 0.0131 and standard deviation of 0.0065 .

ments. However, the absolute thickness determinations may be biased by the geometry of the particular rhabdolith specimen used for calibration. In comparison, use of different rhabdolith specimens for calibration for different measurement sessions could lead to significant variations in thickness results with both poor reproducibility and poor accuracy. If our population is representative, statistically measurement of at least 18 individual rhabdolith specimens in each session would be required to reduce the standard error of the mean (calibration slope) to $10 \%$. Van de Locht et al. (2014) found, using electron tomography, that Rhabdosphaera rods may have a hollow space. This morphology could provide an explanation for the divergent results of Rhabdosphaera.

\section{Application to thickness measurements in the color range}

\subsection{Use of calcite wedge to develop calibration equations in color ranges}

Whereas small placoliths of the modern and late Pleistocene oceans possess coccoliths thinner than $1.55 \mu \mathrm{m}$, in the grayscale range the large modern and ancient placoliths possess thicker coccoliths which enter into interference colors. For this reason, it is fundamental to develop approaches for quantifying thickness beyond the grayscale range. Due to the high density of defects in the color region of OVD-W1, the ETH-W2 was employed for calibration of the relationship between thickness and interference colors. A color image of the calcite wedge was obtained, ensuring that light intensity and camera exposure time were adjusted to give light saturation at the previously calculated point where the wedge reaches $1.55 \mu \mathrm{m}$ of thickness. For the development of calibration equations, 1 out of every 10 pixels was sampled along the calibration profile, leaving ample independent data for subsequent validation.

Digital images from color cameras encode color variations using an additive synthesis of three primary light colors, or RGB. The "Color Transformer" plugin from ImageJ_Fiji was employed to convert RGB values along the calibration profile to two alternative color models: HSV (Fig. 6) and HSL. To identify useful regressions between the components of these color models and thickness, the correlation between the wedge thickness and each of these individual color components was calculated as well as combinations of them, over the entire wedge and over discrete sectors $(0.5 \mu \mathrm{m}$ wide) of the wedge (Table 1). We found slightly higher correlations in the HSV color model than in the HSL, and subsequently used HSV. Although for any given sector of the wedge, there is a color component highly correlated with thickness, no single color component maintains a constant and high sign of correlation with thickness over the entire wedge profile. Therefore, the development of a model to relate thickness to the color components will require (1) alternation between a series of equations, and (2) definition of criteria that uniquely specify, based on the observed color 

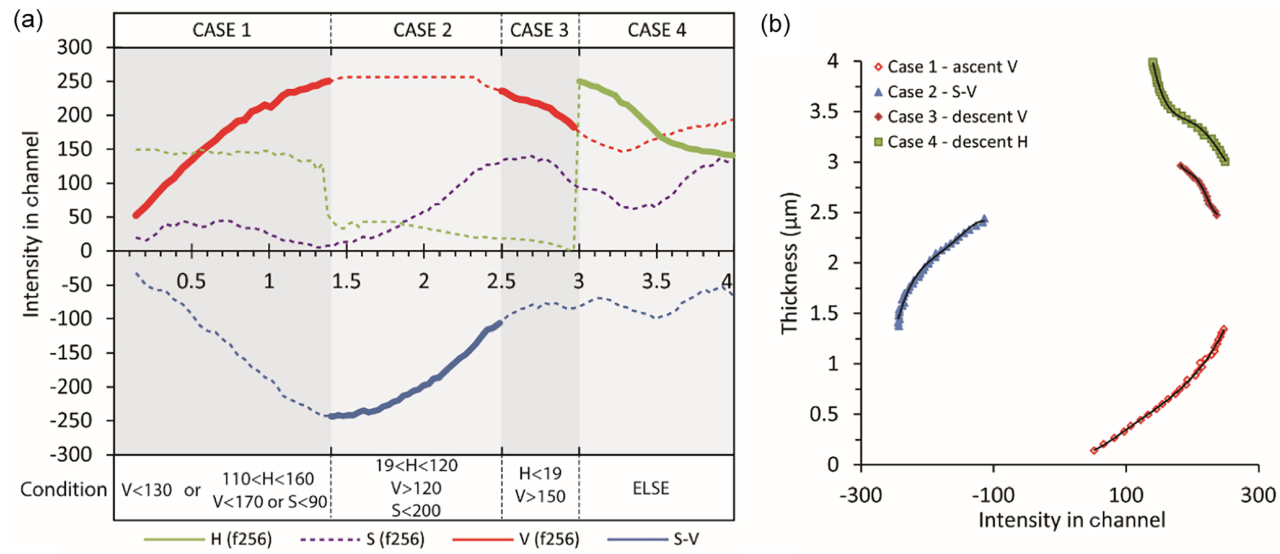

(c)

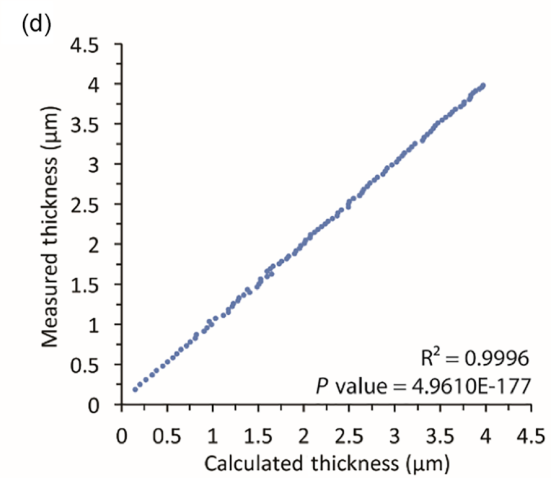

Figure 6. (a) Variation in components of color-model HSV with increasing thickness along the calcite wedge profile. Solid lines indicate the range in which that color component is used to calculate thickness. (b) Polynomial distribution for each interval. Table 2 gives the equations of each color component. (c) Tridimensional Thickness plots of calcite wedge area generated with image analyzer software using criteria and equations described in the text. (d) Validation profile of the measured thickness of the calcite wedge ETH-W2 and the thickness calculated using the regression equations described in Table 2. For the validation data, we used a different set of pixels than those used for the calibration equations. Light intensity was saturated at a calcite thickness of $1.55 \mu \mathrm{m}$.

components, which equation should be used. The definition of these criteria or thresholds is the most challenging part of successfully applying equations to calculate thickness.

From the variation of color components in our calcite wedge, we found that between 0 and $4 \mu \mathrm{m}$, four distinct cases each with their own equation for thickness, can be defined (Fig. 6a; Table 2). The first case is defined as pixels meeting either $V$ values below 130, or $H$ values between 110 and 160 while $S<80$ or $V<170$. It encompasses the thickness range between 0 and $1.4 \mu \mathrm{m}$, over which the $V$ index increases with increasing thickness (positive correlation with thickness of 0.99). The second case is defined by $H$ values between 120 and 19 while $V>120$. It encompasses the thickness range between 1.4 and $2.5 \mu \mathrm{m}$, over which the $S-V$ combination provides optimal positive correlation with thickness (0.96). This component, and all others, shows a lower sensitivity to thickness between 1.5 and $1.7 \mu \mathrm{m}$; the $S-V$ component maintains the best sensitivity to thickness in the range 2.2 to $2.4 \mu \mathrm{m}$. A third case is defined by $H<19$ and $V>150$. This encompasses the thickness range from 2.5 to $3 \mu \mathrm{m}$, over which the $V$ index shows a high negative correlation with thickness (0.98). A final interval is defined for all pixels not meeting the criteria of the other three categories. This interval includes the thickness range 3-4 $\mu \mathrm{m}$, over which the $H$ index shows a strong and negative linear correlation with thickness (0.97). While the correlation table shows linear regressions, the best fits are in some cases polynomials (Fig. 6b). Equations derived from these polynomial fits are ultimately used for the calculation of thickness from the various color indexes, with attention paid to employing at least five significant figures in the coefficients of the equations to prevent rounding errors.

This calibration has been validated by comparing the observed and calculated thickness of pixels along the calibration line of the ETH-W2 calcite wedge, using pixels which were not employed to generate the regression equations (Fig. 6c). Results show a continuously robust estimation of thickness throughout the entire range from 0 to $4 \mu \mathrm{m}$. Using open-source software ImageJ-Fiji, we analyzed images of both wedge profile regions and applied the conditions and equations to validate the thickness with the birefringence color (Fig. 6d). 


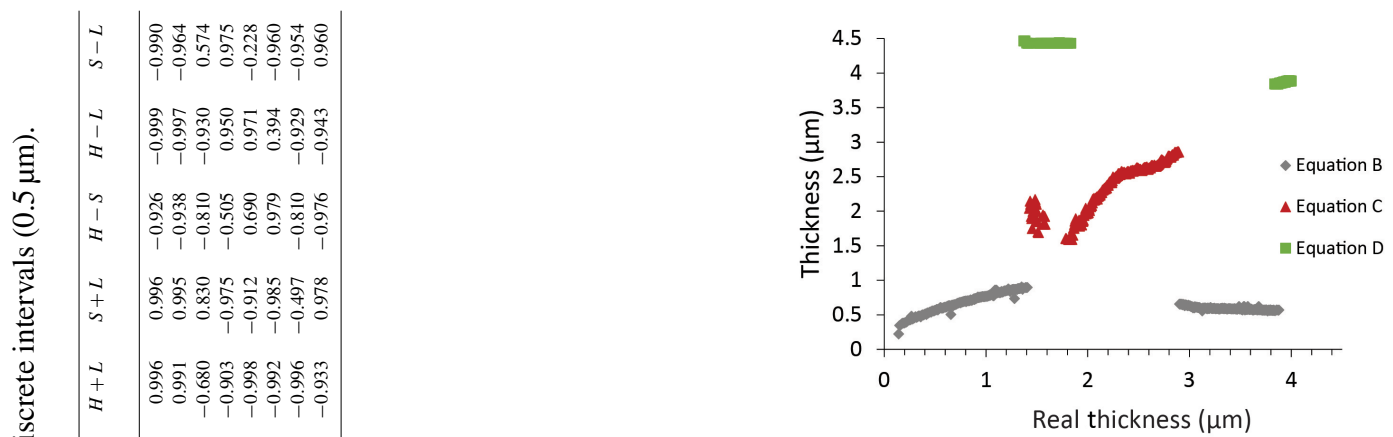

Figure 7. Relationship between measured thickness (with tilting compensator) of the ETH-W2 calcite wedge and thickness calculated by application of the equations of Beaufort et al. (2014) for calculation of calcite thickness from HSL color model, for the validation dataset from the calcite wedge. The colors indicate the equation specified for use according to the criteria of Beaufort et al. (2014).

These particular equations may not be exportable to other different microscopy and camera systems, or other camera or light configurations on a same microscope. For example, a previous publication (Beaufort et al., 2014) proposes a set of three equations employing the HSL index for calculation of thickness values between 0 and $4.5 \mu \mathrm{m}$. Using the validation dataset from our profile along the calcite wedge, thickness was calculated using the exact equations and thresholds provided by Beaufort et al. (2014). The thickness calculated from those equations deviates significantly from the actual calcite wedge thickness along several portions of the calcite wedge, in particular in the ranges 1.4-1.7 and 3$4 \mu \mathrm{m}$ (Fig. 7). This result shows that both the thresholds and equations relating color to thickness must be calibrated on each microscope system and settings. We suggest that several factors may cause variation in the color components for a given thickness. Firstly, the spectrum of the microscope light source will vary in intensity at different color wavelengths, and this may vary both among microscopes and over time due to bulb aging. Secondly, the use of filters as well as objective characteristics, diaphragm aperture, light intensity, and light absorption by slides within the microscope system, may affect the color components for a given thickness. Also, another major source of variation is the color transformation done by different color-scale cameras, which should be corrected to decrease variations between different microscope setups (Johnsen et al., 2017). Finally, within the digital camera, the quantum efficiency for a given wavelength may be different for different camera detectors. For example, the Zeiss camera employed here reports a very high efficiency in the green region compared to red or blue, whereas other cameras report a more similar efficiency among the wavelengths. Adjustments in the white balance in the image analysis system can in theory make the color components more similar among different cameras, but in practice we have found that 
there is still variation that requires adjustment to the calibration. Even small variations in the calibration equations become very important in the estimation of thickness in coccoliths, as described in Sect. 5.2.

Since the relationship between interference colors and thickness must be established for each particular microscope-camera configuration and validated routinely, we propose that a calcite wedge may serve as one such optimal calibration material. For a new microscope system, we advise the capture of a digital color image of a calcite wedge for which the profile of thickness has been independently determined by the tilting compensator. One of the first steps would be to graphically represent thickness and color components to determine zones of useful relationships between them. A second step would be to identify the unique thresholds which could be used to distinguish portions of the thickness range requiring different equations. In HSV, one would expect a similar basic sequence of changes in color parameters with increasing thickness; however, the exact values of the threshold parameters can change appreciably with small changes in microscope or camera settings. The most significant challenge of calibration lies in identifying the thresholds, because errors in threshold definition result in application of the inappropriate equation for calculating thickness for that pixel and thus lead to large errors in estimates of thickness in coccoliths. The saturation of light at a thickness of $1.55 \mu \mathrm{m}$ is also crucial since it allows the boundary to be set between the gray domain (black to white) and the color range and can be verified either with synthetic polymers or prior mapping of the zone of such thickness on the calcite wedge. In our experience, it is important to define saturation as the first appearance of grayscale values of 255 in the digital image (taken with an 8-bit camera), rather than relying on the mapping of saturation by the image analysis system. Oversaturated images can produce blank areas and peaks in $H$ values between the thresholds for our case 1 and case 3, which leads to an overlap in criteria for thicknesses in the 1.3 to 1.4 and 3.2 to $3.3 \mu \mathrm{m}$ thickness ranges, and misapplication of the case 4 equation to pixels in the 1.3 to $1.4 \mu \mathrm{m}$ thickness range.

\subsection{Application to ancient Reticulofenestra specimens and modern Helicosphaera carteri coccoliths}

In order to test calibration equations and thresholds, a macro in ImageJ-Fiji has been implemented to automate the calculation of the thickness of coccoliths as well as geometric parameters. This tool reads pixel values from a selected specimen and applies the appropriate regression equations to each pixel, creating a thickness map of the coccolith.

For this test, we have observed slides settled following the method developed by Flores and Sierro (1997). Samples from the IODP Expedition 342 were used because the preservation of coccolithophores is moderate to good (Norris et al., 2014). Moreover, the coccolith thickness in some

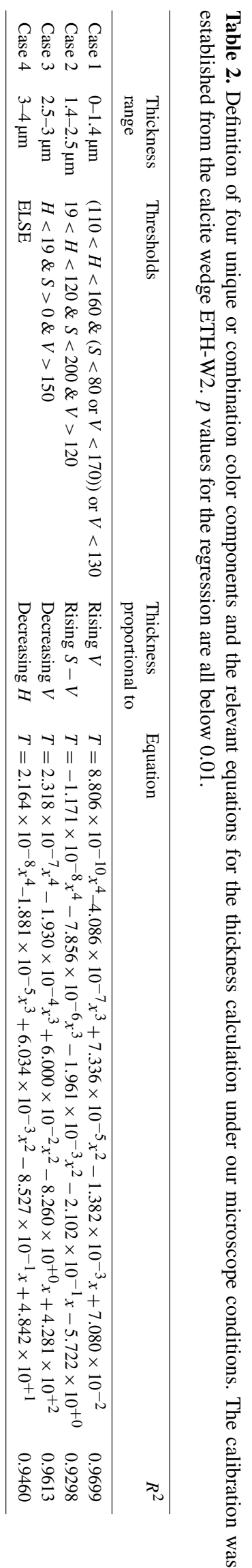

www.biogeosciences.net/15/1079/2018/ 

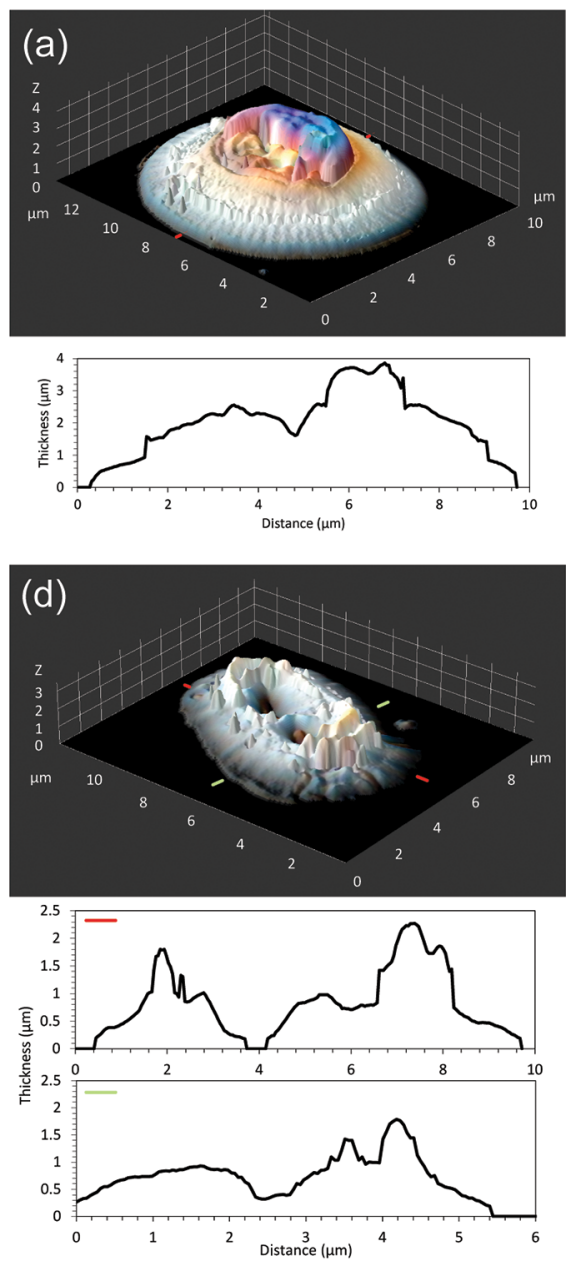
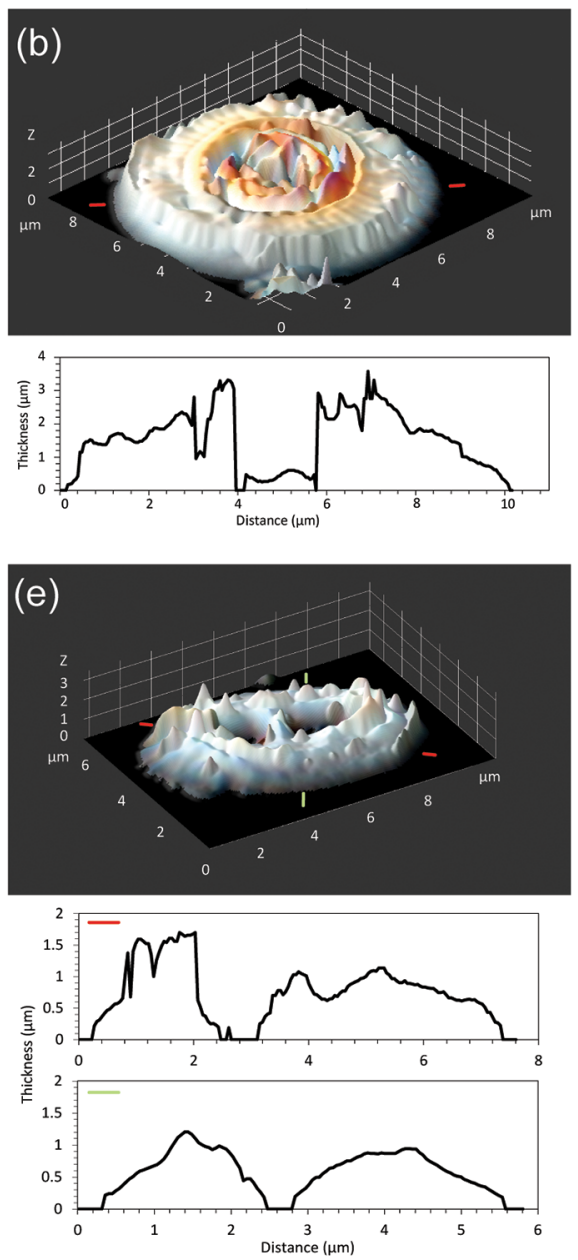
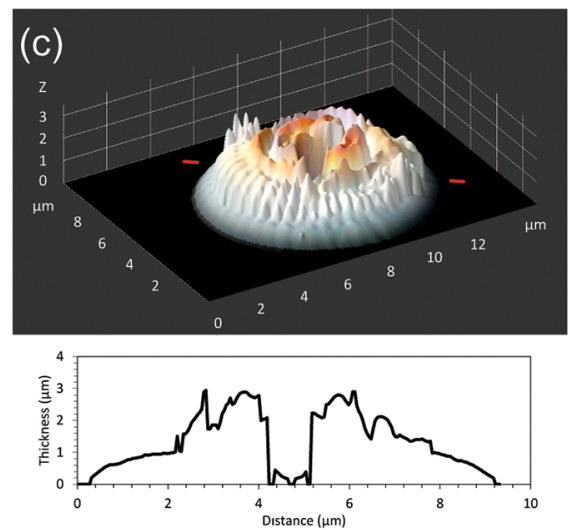

(f)
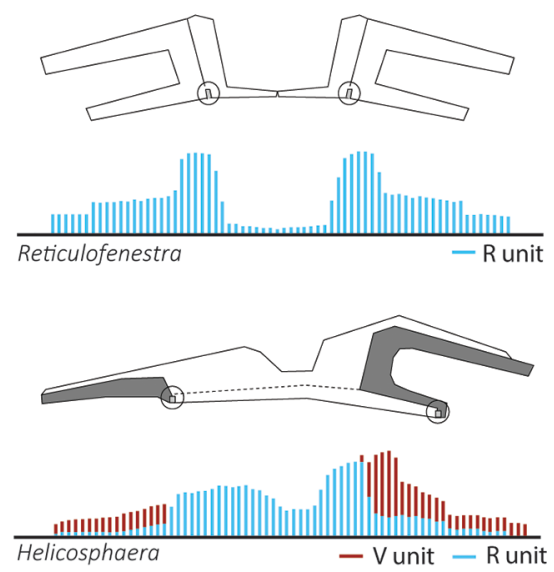

Figure 8. Sample of accumulated thickness schemes overlapped with color images and cross-section profiles for Reticulofenestra bisecta (a), Cyclicargolithus floridanus (b-c) from U1406A, and Helicosphaera carteri (d-e) from 905. (f) Thickness representation of two main coccolithophore groups used here from its cross-section shape schemes modified from Young et al. (2003).

parts of the Oligocene sequence exceeds $1.55 \mu \mathrm{m}$. We have complemented this check with Zygodiscales Helicosphaera carteri taxa of the late Quaternary age from the NIOP 905 site.

Several sample thickness maps and profiles for Reticulofenestra spp. and $H$. carteri individuals are shown in Fig. 8a-e. Tridimensional schemes represent total thickness accumulated across the coccolith crystal units for which the optical axis is not parallel to the light ray that is with radially oriented axes. Essentially all of the Reticulofenestra calcite is radially oriented and thus effectively quantified. In contrast, the $H$. carteri coccoliths feature both radially oriented and vertically oriented calcite, of which the thickness of the radially oriented calcite is accurately calibrated by the calcite wedge equations. Although the surface plot is not smooth, the cross-section profiles show a pattern that can be coherently related to the main thickness configuration plots (Fig. 8f), built from the models of Young et al. (2003). From such maps, the mean coccolith thickness may be calculated and permits estimates of evolution of calcification of large coccolith species over time and in different locations.

\section{Useful online resources}

The complementary web information about microscopy techniques, polarization, coccolithophore species, digital imaging, and image processing that has been consulted during this work can be located via the following links:

- http://fiji.sc/Fiji

- http://www.microscopy-uk.org.uk/mag/artmay14/ jp-retardation.pdf

- http://www.microscopyu.com/

- http://www.mikrotax.org/Nannotax3/ 
- http://www.modernmicroscopy.com/

- http://zeiss-campus.magnet.fsu.edu/
Edited by: Marcel van der Meer

Reviewed by: Xiaobo Jin and one anonymous referee

\section{Conclusions}

The ability of the scientific community to compare optically determined estimates of coccolith thickness among different laboratories will depend on the reproducibility of the methods for calibration of relationships between thickness and light intensity. A detailed survey of the behavior of one commonly used calibration material, $R$. clavigera, showed that the calibration is highly dependent on the particular calibration specimen used. In addition, calibrations in the 1.55 to $4 \mu \mathrm{m}$ thickness range require a calibration material to accurately account for differences in color spectra under different measurement conditions. We propose a new calibration method based on the development and use of a wedge of calcite to relate light intensity recorded with the coccolith thickness. The thickness of the calcite wedge can be independently calibrated using a tilting compensator and validated using polymers of known retardation. The calcite wedge calibration has been applied to the measurement of thickness of cultured coccoliths from several strains of coccolithophorids. This allowed an examination of specimens to quantify the $>1.55 \mu \mathrm{m}$ thickness the birefringence of which spans beyond the grayscale into the color range. In the future, the use of image analysis software could permit the development of macros to further automate the calibration process.

Data availability. No data sets were used in this article.

Author contributions. This study was conceived by HMS. Measurements were conducted by SGL and JG, with guidance from JAF and MAF. Calculations and analysis were conducted by SGL, HMS, and JG; SGL, JG, and HMS wrote the paper, with input from other authors.

Competing interests. The authors declare that they have no conflict of interest.

Acknowledgements. This research was funded by the European Research Council under grant ERC-STG-240222 PACE to Heather M. Stoll. We thank Andrés Cuesta and Ángel R. Rey for advice on the manufacture of the calcite wedge and use of the Berek and tilting compensators, and Ángel M. Nistal for assistance in macro development in ImageJ-Fiji. We thank Xiaobo Jin, Simen Alexander Linge Johnsen, Jörg Bollman, and one anonymous reviewer as well as the editor for their comments and suggestions, which helped us to improve the original version of the paper.

\section{References}

Abrantes, F., Meggers, H., Nave, S., Bollman, J., Palma, S., Sprengel, C., Henderiks, J., Spies, A., Salgueiro, E., Moita, T., and Neuer, S.: Fluxes of micro-organisms along a productivity gradient in the Canary Islands region $\left(29^{\circ} \mathrm{N}\right)$ : implications for paleoreconstructions, Deep-Sea Res. Pt. II, 49, 3599-3629, https://doi.org/10.1016/S0967-0645(02)00100-5, 2002.

Armstrong, R. A., Lee, C., Hedges, J. I., Honjo, S., and Wakeham, S. G.: A new, mechanistic model for organic carbon fluxes in the ocean based on the quantitative association of POC with ballast minerals, Deep-Sea Res. Pt. II, 49, 219-236, https://doi.org/10.1016/S0967-0645(01)00101-1, 2002.

Armstrong, R. A., Peterson, M. L., Lee, C., and Wakeham, S. G.: Settling velocity spectra and the ballast ratio hypothesis, Deep-Sea Res. Pt. II, 56, 1470-1478, https://doi.org/10.1016/j.dsr2.2008.11.032, 2009.

Bárcena, M. A., Flores, J. A., Sierro, F. J., Pérez-Folgado, M., Fabres, J., Calafat, A., and Canals, M.: Planktonic response to main oceanographic changes in the Alboran Sea (Western Mediterranean) as documented in sediment traps and surface sediments, Marine Micropaleontol., 53, 423-445, https://doi.org/10.1016/j.marmicro.2004.09.009, 2004.

Bass, M.: Handbook of optics, 3rd Edn., McGraw-Hill, 2009.

Beaufort, L.: Weight estimates of coccoliths using the optical properties (birefringence) of calcite, Micropaleontology, 51, 289297, https://doi.org/10.2113/gsmicropal.51.4.289, 2005.

Beaufort, L. and Dollfus, D.: Automatic recognition of coccoliths by dynamical neural networks, Mar. Micropaleontol., 51, 57-73, 2004.

Beaufort, L., Barbarin, N., and Gally, Y.: Optical measurements to determine the thickness of calcite crystals and the mass of thin carbonate particles such as coccoliths, Nat. Protoc., 9, 633-642, https://doi.org/10.1038/nprot.2014.028, 2014.

Berger, W. H., Smetacek, V., and Wefer, G.: Ocean productivity and paleoproductivity-an overview, Productivity of the Oceans present and past: Report of the Dahlem Workshop on Productivity of the Ocean, Berlín, 1-34, 1989.

Bollmann, J.: Technical Note: Weight approximation of coccoliths using a circular polarizer and interference colour derived retardation estimates - (The CPR Method), Biogeosciences, 11, 18991910, https://doi.org/10.5194/bg-11-1899-2014, 2014.

Bolton, C. T., Hernández-Sánchez, M. T., Fuertes, M. Á., González-Lemos, S., Abrevaya, L., Mendez-Vicente, A., Flores, J.-A., Probert, I., Giosan, L., Johnson, J., and Stoll, H. M.: Decrease in coccolithophore calcification and $\mathrm{CO}_{2}$ since the middle Miocene, Nat. Commun., 7, 10284, https://doi.org/10.1038/ncomms10284, 2016.

Craig, D. B.: The Benford plate, Am. Mineral., 46, 757-758, 1961. Delly, J. G.: The Michel-Lévy interference color chart - microscopy's magical color key, available at: https://www.mccrone.com/mm/the-michel-levy-interferencecolor-chart-microscopys-magical-color-key/ (last access: 14 February 2018), 2003. 
Engel, A., Abramson, L., Szlosek, J., Liu, Z., Stewart, G., Hirschberg, D., and Lee, C.: Investigating the effect of ballasting by $\mathrm{CaCO}_{3}$ in Emiliania huxleyi, II: Decomposition of particulate organic matter, Deep-Sea Res. Pt. II, 56, 1408-1419, https://doi.org/10.1016/j.dsr2.2008.11.028, 2009.

Flores, J. A. and Sierro, F. J.: Revised technique for calculation of calcareous nannofossil accumulation rates, Micropaleontology, 43, 321-324, https://doi.org/10.2307/1485832, 1997.

Frohlich, M. W.: Birefringent objects visualized by circular polarization microscopy, Stain Technol., 61, 139-143, 1986.

Fuertes, M. Á., Flores, J. A., and Sierro, F. J.: The use of circularly polarized light for biometry, identification and estimation of mass of coccoliths, Mar. Micropaleontol., 113, 44-55, https://doi.org/10.1016/j.marmicro.2014.08.007, 2014.

Hecht, E.: Optics, 4th. International Edn., Addison-Wesley, San Francisco, Vol. 3, 2002.

Higgins, M. D.: Imaging birefringent minerals without extinction using circularly polarized light, Can. Mineral., 48, 231-235, https://doi.org/10.3749/canmin.48.1.231, 2010.

Iversen, M. H. and Ploug, H.: Ballast minerals and the sinking carbon flux in the ocean: carbon-specific respiration rates and sinking velocity of marine snow aggregates, Biogeosciences, 7 , 2613-2624, https://doi.org/10.5194/bg-7-2613-2010, 2010.

Johnsen, L. S., Bollmann, J., Lee, H. W., and Zhou, Y.: Accurate representation of interference colours (Michel-Lévy chart): from rendering to image colour correction, J. Microsc.-Oxford, https://doi.org/10.1111/jmi.12641, online first, 2017.

Lochte, A. A.: Single coccolith weight estimates using two birefringence methods on cultured Gephyrocapsa oceanica, MsC, Uppsala, Sweden, 2014.

Milliman, J. D.: Production and accumulation of calcium carbonate in the ocean: budget of a nonsteady state, Global Biogeochem. Cy., 7, 927-957, https://doi.org/10.1029/93GB02524, 1993.
Norris, R., Wilson, P., Blum, P., and ExpeditionScientists: Proceedings IODP, 342, College Station, TX (Integrated Ocean Drilling Program), https://doi.org/10.2204/iodp, 2014.

O’Dea, S. A., Gibbs, S. J., Bown, P. R., Young, J. R., Poulton, A. J., Newsam, C., and Wilson, P. A.: Coccolithophore calcification response to past ocean acidification and climate change, Nat. Commun., 5, 5363, https://doi.org/10.1038/ncomms6363, 2014.

Ridgwell, A. and Zeebe, R. E.: The role of the global carbonate cycle in the regulation and evolution of the Earth system, Earth Planet. Sc. Lett., 234, 299-315, https://doi.org/10.1016/j.epsl.2005.03.006, 2005.

Siesser, W. G.: Calcareous nannoplankton, in: Fossil Prokaryotes and Protists, edited by: Lipps, J. H., Blackwell Scientific Publications, Boston, 169-201, 1993.

Sørensen, B. E.: A revised Michel-Lévy interference colour chart based on first-principles calculations, Eur. J. Mineral., 25, 5-10, https://doi.org/10.1127/0935-1221/2013/0025-2252, 2013.

van de Locht, R., Slater, T. J., Verch, A., Young, J. R., Haigh, S. J., and Kröger, R.: Ultrastructure and crystallography of nanoscale calcite building blocks in Rhabdosphaera clavigera coccolith spines, Cryst. Growth Des., 14, 1710-1718, https://doi.org/10.1021/cg4018486, 2014.

Young, J. R., Geisen, M., Cros, L., Kleijne, A., Sprengel, C., Probert, I., and Østergaard, J.: A guide to extant coccolithophore taxonomy, Journal of Nannoplankton Research, 1, 1-125, 2003.

Ziveri, P., de Bernardi, B., Baumann, K.-H., Stoll, H. M., and Mortyn, P. G.: Sinking of coccolith carbonate and potential contribution to organic carbon ballasting in the deep ocean, Deep-Sea Res. Pt. II, 54, 659-675, https://doi.org/10.1016/j.dsr2.2007.01.006, 2007. 